\title{
Efficient Fronthaul and Backhaul Connectivity for IoT Traffic in Rural Areas
}

\author{
Elias Yaacoub ${ }^{1}$ and Mohamed-Slim Alouini ${ }^{2}$ \\ ${ }^{1}$ Dept. of Computer Science and Engineering, College of Engineering, Qatar University (QU), Doha, Qatar. \\ ${ }^{2}$ Computer, Electrical and Mathematical Sciences and Engineering (CEMSE) Division, King Abdullah University of Science and \\ Technology (KAUST), Thuwal, Makkah Province, Saudi Arabia. \\ Emails: eliasy@ieee.org, slim.alouini@kaust.edu.sa
}

\begin{abstract}
In this paper, internet of things (IoT) connectivity in rural areas is investigated. Both fronthaul and backhaul considerations are studied. First, intelligent radio resource management (RRM) and network planning techniques are discussed for IoT access/fronthaul networks. The proposed RRM scheduling approach was shown to lead to good performance in scheduling IoT devices. Then, several backhauling techniques for providing connectivity to rural areas are investigated and their cost efficiency is analyzed. Techniques based on free space optics with solar powered devices are found to be a suitable backhaul solution.
\end{abstract}

\section{INTRODUCTION}

The internet of things (IoT), with its billions of devices sensing every aspect of our daily lives, is considered as the backbone of smart cities [1]. There are a lot of applications where IoT is at the heart of smart cities, e.g., smart grid [2], intelligent transportation systems, environment protection [3], water resources monitoring and management, and smart healthcare, among others.

All these systems rely on accurate and ubiquitous connectivity, thus necessitating the deployment of efficient communication networks. From the fiber infrastructure to 5G and beyond wireless access, communication networks need to support the load of increased IoT machine-to-machine (M2M) communications, under the umbrella of massive machine type communications (mMTC) in the 5G standards, in addition to the tremendous bandwidth demand in traditional communications (data, video, etc.), falling under the scenario of enhanced mobile broadband (eMBB) in the 5G standards. Consequently, they must satisfy the stringent requirements of certain M2M applications (smart grid, m-Health), while maintaining the quality of service (QoS) and quality of experience (QoE) of network subscribers. Furthermore, it is to be noted that this enhanced network operation should take into account energy efficiency and the power consumption of the communication networks. Therefore, several techniques come into play to reach this target: software defined networking and smart network slicing, coupled with green networking techniques, inter-operator collaborations, and the use of cellular base stations powered by renewable energy.

It can be argued that that the above technological advances, although necessary for a smart city, can enable smart living in general (e.g., smart villages, smart suburbs). However, to ensure their successful implementation in rural areas outside cities, not only efficient IoT deployments should take place, but also the deployment of an adequate backhaul network relaying the rural IoT traffic to the network core is essential.

Therefore, this paper addresses the problem of IoT deployments in rural areas. First, we present a radio resource management approach (RRM) that can adapt to various IoT traffic densities. Although this approach is applicable to both urban and rural areas, it is particularly suited to IoT deployments in areas with limited spectrum resources. Then, we address the problem of providing backhaul connectivity for the rural IoT traffic. Indeed, the main challenge is in ensuring fast, reliable connectivity in rural areas supporting the needed IoT traffic to enable a smart living environment. Hence, we discuss several technological solutions for rural backhaul, and analyze their cost efficiency taking into account capital expenditures (CAPEX) and long-term operational expenditures (OPEX).

\section{From SMART CitTIES TO SMART LIVING}

Smart city advocates aim to manage the problem of having half the world population already living in cities and another couple billion being on their way. As stated in [4], "the world is becoming far more urbanized, and mega cities with populations greater than ten to twenty million people are emerging, there is a greater need for large-scale operations and management for cities to effectively serve its inhabitants."

However, with the expected technological advances, especially the ones based on IoT, we can have: smart villages, smart towns, smart suburbs, etc. One can enjoy quality healthcare, quality education, and several jobs can tolerate employees working remotely with all the progress in communications technologies. All this can take place in a less crowded, less polluted rural environment. Thus, quality living can be enjoyed without having to move to a big city.

Imagine you live in a "smart home" in a quiet, clean village, with solar panels generating energy to (at least partially) meet the consumption demands; you work from home, where you are connected through a high bandwidth communications network to your colleagues, whether they are 
also working from home or at the company's headquarters; your kids enjoy distance learning through technology and VR methods; you can enjoy fresh organic food due to smart agriculture; you can rely on a high speed rail network whenever you (occasionally) need to go to the city; quality healthcare is available at a local health center, with even complicated surgeries being possible with the help of robotics, augmented reality (AR) / virtual reality (VR), and ultrareliability low latency communications (URLLC) in 5G networks. Would you need to live in a crowded, polluted city just to benefit from the same advantages?

Hence, while the technological progress has made the existence of smart cities with huge populations possible, that same progress also made it possible for people to enjoy quality living in their rural areas. This "smart everywhere" concept, coupled with smart governance, can lead to a more balanced population density between cities and rural areas, while allowing all citizens to enjoy quality living. However, while the deployment of IoT devices can take place in both rural and urban areas, the "smart everywhere" scenario depends on high speed connectivity between the IoT devices and the core network. This can be performed in a profitable manner in cities and densely populated areas. However, the challenge is in providing high speed backhaul connectivity to rural areas [5].

In addition, when discussing connectivity in rural areas, there must be a differentiation between:

- Remote areas in developed countries, where transportation networks (e.g., rail) and electricity are provided, and

- Poor areas in developing countries, where the basic services are hardly provided to the population.

Rural Backhaul in Developed Countries:

Generally, these rural areas are accessible by transportation networks, e.g., railroad networks. Furthermore, power is provided through the electricity grid. The challenges in providing connectivity to these areas lie in the difficulty of obtaining a good return on investment (ROI) for mobile operators. A possible solution is that fiber optic cables can be deployed along the railroad track, thus benefiting from the existing transportation infrastructure. Base station (BS) towers can be erected near the train stops corresponding to population agglomerations in nearby villages/towns. In addition, microwave links can be used for backhaul connectivity by reusing the existing towers used for deploying GSM-R BSs to maintain connectivity for operating the train system. Fiber can also be deployed at lower costs by using micro-trenching on the border of roadways [6]. Access technologies can then be implemented as needed, whether traditional methods, or novel ones, e.g., Terragraph, a $60 \mathrm{GHz}$ multi-hop multi-point wireless distribution network [7].

Thus, in these areas, Access/Fronthaul connectivity can be provided within each town/village using the appropriate technology, then the access BSs can be connected to the backbone network through the microwave or fiber systems deployed along the transportation routes.

\section{Rural Backhaul in Developing Countries:}

This scenario corresponds to poor areas in developing countries where the challenge is to reduce the digital divide with developed countries. Hence, connectivity needs to be provided to secure basic services, such as healthcare, education, basic web access, etc. In these areas, transportation roads are under-developed or even non-existent, and the villages are almost isolated from the major cities within their country, e.g., see [8]. Power generation could rely on local generators, or on a small local grid.

Hence, providing backhaul connectivity to these rural areas cannot benefit from existing infrastructure, and has often to be done from scratch. Furthermore, the poverty of the people living there, even regardless of the population density, does not promise sufficient revenues to justify the backhaul deployment costs of a mobile operator. State subsidies can be provided to support deployment, which nevertheless should be as cost efficient as possible.

Therefore, analyzing the costs of providing backhaul solutions for this kind of rural areas is one of the two main objectives of this paper. In the next section, we discuss the first main objective of providing a fronthaul/access approach for IoT devices that can be adapted dynamically to population density, then, in the following section, we tackle the second main objective by investigating in more detail rural backhaul costs and suitable solutions.

\section{AN RRM APPROACH FOR IOT ACCESS/FRONTHAUL}

In this section, we present a smart RRM approach, coupled with an intelligent network planning approach, that allows IoT devices to access the network and transmit their data. The proposed approach allows the accommodation of varying densities of IoT devices and thus can be used in urban and rural areas. Although the proposed RRM approach is analyzed in a simulation environment based on LTE-Advanced-Pro Narrowband-IoT (NB-IoT) and on scheduling using proportional fair in time and frequency (PFTF) as proposed in [2], it can be easily extended to other technologies by replacing LTE resource blocks (RBs) with the resources that can be allocated in a given technology.

\section{A. RRM Algorithm}

This section presents the RRM algorithm for IoT traffic. In LTE, each transmission time interval (TTI) has a duration of 1ms. In addition, orthogonal frequency division multiple access (OFDMA) is used. In each TTI, the smallest allocated resource is a group of 12 OFDMA subcarriers, forming an 
LTE resource block (RB). We assume that each IoT device has to achieve an average target data rate $\mathrm{R}_{\mathrm{T}}$, during a certain time interval, corresponding to a number of $\mathrm{N}_{\text {TTI }}$ TTIs.

A high-level summary of the RRM algorithm can then be presented as follows:

1: For each TTI (going from 1 to $\mathrm{N}_{\mathrm{TTI}}$ )

2: Consider the set of available RBs for IoT traffic

3: Consider the set of active IoT devices in the current period

4: $\quad$ For each RB

5: $\quad$ Find the device that, when allocated that RB can achieve the highest proportional increase in its data rate, compared to its data rate before being allocated that $\mathrm{RB}$

6: $\quad$ Allocate the RB to that device

7: $\quad$ Remove the RB from the set of available RBs

8: $\quad$ If the device can meet its target rate on time with its allocated RB(s)

9:

Remove the device from the set of available IoT devices (i.e., those requesting resources)

10: $\quad$ Else

11: $\quad$ Keep the device in the set of available IoT devices

12: $\quad$ End

13: End

14: End

\section{B. Simulation Results}

This section presents the simulation results using the RRM algorithm of the previous section. We consider a cell of $500 \mathrm{~m}$ radius. The simulation parameters concerning transmit power and the channel model are used as in [2]. Fig. 1 shows the outage rate versus the number of simultaneously active IoT devices, for different numbers of available RBs and different target rates $R_{T}$. Fig. 1 (a) shows that when $R_{T}$ decreases, the number of IoT devices that can be simultaneously served, for a given outage rate, increases significantly. Furthermore, it shows the expected result that as the number of RBs dedicated for IoT traffic increases, the number of served IoT devices also increases significantly.

To get more insights on how to use these results for dimensioning $5 \mathrm{G}$ networks to handle IoT traffic, Fig. 1(b) shows a detailed view of Fig. 1(a), with horizontal lines indicating $0.2 \%$ and $0.5 \%$ outage, respectively. For example, 2000 IoT devices can be served simultaneously with an outage rate below $0.5 \%$ if 50 RBs are available, for a target rate $R_{T}$ $=20 \mathrm{kbps}$. Also, around 300 IoT devices can be served simultaneously with an outage rate below $0.2 \%$ if $25 \mathrm{RBs}$ are available, for a target rate $\mathrm{R}_{\mathrm{T}}=60 \mathrm{kbps}$.

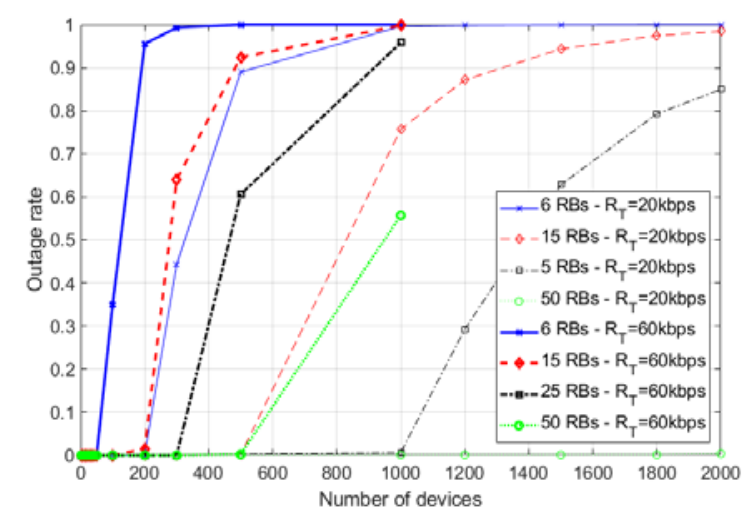

(a)

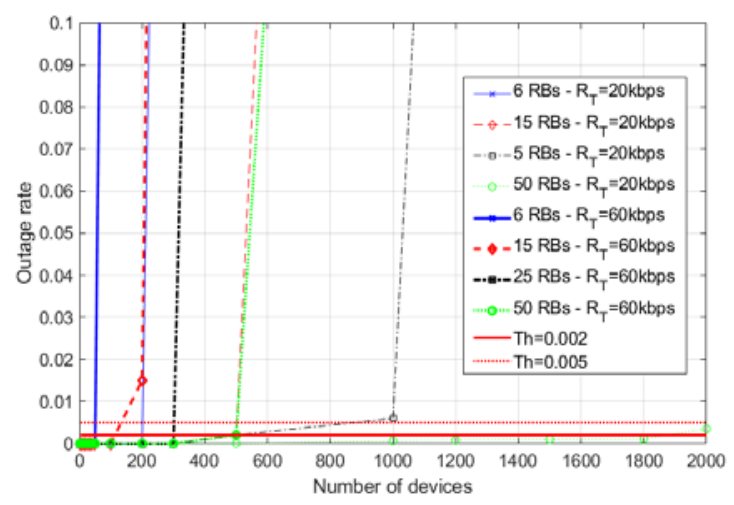

(b)

Figure 1. Outage results using the proposed RRM algorithm: (a) Outage results; (b) Zoomed-in view on Fig. 1(a).

\section{Planning IoT Access Networks}

Table 1. IoT Network Planning Example for $\mathrm{R}_{\mathrm{T}}=60 \mathrm{kbps}$, and Each Device Transmitting for 500ms every 120 s.

\begin{tabular}{|c|c|}
\hline \multicolumn{2}{|c|}{ With $\mathbf{N}_{\mathrm{RB}, \mathrm{LR}}=\mathbf{5 0}$ and $\mathrm{N}_{\mathrm{RB}, \mathrm{RS}}=\mathbf{1}$} \\
\hline $\begin{array}{c}\text { Number of Relays } \\
\left(\mathrm{N}_{\mathrm{RS}}\right)\end{array}$ & $\begin{array}{c}\text { Number of Devices } \\
\text { served per Relay }\left(\mathrm{N}_{\mathrm{D}, \mathrm{RS}}\right)\end{array}$ \\
\hline $\mathrm{N}_{\mathrm{RS}}=500$ & $\mathrm{~N}_{\mathrm{D}, \mathrm{RS}}=240$ \\
\hline $\mathrm{N}_{\mathrm{RS}}=50$ & $\mathrm{~N}_{\mathrm{D}, \mathrm{RS}}=2400$ \\
\hline $\mathrm{N}_{\mathrm{RS}}=100$ & $\mathrm{~N}_{\mathrm{D}, \mathrm{RS}}=1200$ \\
\hline $\mathrm{N}_{\mathrm{RS}}=250$ & $\mathrm{~N}_{\mathrm{D}, \mathrm{RS}}=480$ \\
\hline
\end{tabular}


This section presents insights on planning the IoT access network using the results of Fig. 1. For example, as stated in [2] and the references therein for real-time smart meter readings, data transmission by a smart meter occurs every $\mathrm{T}_{\text {period }}=120 \mathrm{~s}$, where the meter performs a transaction by accessing the channel for a duration of $\mathrm{N}_{\mathrm{TTI}}=500 \mathrm{~ms}$, to transmit the meter's data at a rate of $\mathrm{R}_{\mathrm{T}}=60 \mathrm{kbps}$. Thus, for every $\mathrm{N}_{\text {TTI }}=500 \mathrm{~ms}$, the number of simultaneously active meters can be derived from Fig. 1(a), for a given $\mathrm{R}_{\mathrm{T}}$ and $\mathrm{a}$ given tolerable outage threshold Th (e.g. 0.2\%). However, since each meter has to access the channel for 500ms during $120 \mathrm{~s}$, then the actual number of served meters can be multiplied by 240 (=120s/0.5s) since they can be scheduled to access the channel in different batches. Consequently, relay stations (RSs)/aggregators can be deployed to serve a group of IoT devices using $\mathrm{N}_{\mathrm{RB}, \mathrm{RS}} \mathrm{RBs}$ on the link between aggregator and devices, with the aggregators transmitting continuously the traffic to a BS using $\mathrm{N}_{\mathrm{RB}, \mathrm{LR}} \mathrm{RBs}$ dedicated to the RS-BS link. With this scenario, each device accesses the channel for

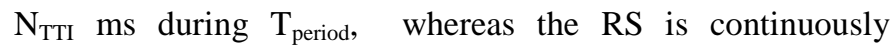
transmitting the aggregated traffic. An example is shown in Table 1.

Form Table 1, the scenario with 500 relays each serving 240 devices corresponds to a sparse deployment, e.g., smart meter deployment in a small rural village. However, the scenario with 50 relays each serving 2400 devices corresponds to a much denser deployment, e.g., in an urban neighborhood. Other examples can be less demanding, e.g., sensor measurements for air pollution monitoring can be received every 5 minutes, or even 15 minutes (instead of 2 minutes as in Table 1), and can be satisfied with $\mathrm{R}_{\mathrm{T}}=20 \mathrm{kbps}$ [3]. This would lead to higher numbers of RSs and devices than shown in Table 1.

Thus, IoT traffic can be served in both urban and rural areas with the state-of-the-art wireless access networks. However, the challenge is in providing backhaul connectivity from the access BSs in rural areas to the core network. This will be the topic of the next section.

\section{OVERVIEW OF RURAL BACKHAUL SOLUTIONS}

In this paper, several solutions for providing backhaul connectivity to rural areas are investigated in terms of their cost, consisting of capital expenditure (CAPEX) and operational expenditures (OPEX). These include fiber, microwave, and free space optics (FSO). The closest study to this paper is in [9], where the costs of fiber optics, microwave links, in addition to terrestrial and vertical FSO are analyzed and compared. However, [9] considers a fronthaul/backhaul scenario, with a dense deployment of BSs, such that 100 macro BSs and 1000 small cell BSs are deployed in an area of size $5 \times 5 \mathrm{Km}^{2}$. Clearly, the results of such a scenario cannot be generalized to a pure backhaul scenario where the objective is to transport the traffic over hundreds of kilometers.
In fact, as mentioned previously, we consider remote rural areas where the backhaul link needs to traverse long distances, without necessarily having any access/fronthaul BSs along the way, before reaching the remote area. Such an area is considered in [8] for example, where 12 hours are needed to reach the area from the nearest urban town. Thus, stretches of hundreds of kilometers could be traversed by the backhaul link before reaching the target area. Therefore, we base our subsequent cost analysis on a distance of $100 \mathrm{Km}$, and we analyze the costs of laying fiber, erecting microwave or terrestrial FSO towers, or using high altitude platforms (HAPs) for "vertical" FSO. Nevertheless, we use the cost parameters defined in [9], and make the necessary adjustments whenever these parameters do not apply to the considered rural scenario, while providing appropriate justifications or references.

Satellite communications are not considered in this paper, mainly for the following reasons: The large delays incurred in satellite communications, the generally large costs for launching a satellite and placing it in orbit, and for fairness considerations. In fact, although satellites can be used to provide backhaul connectivity, satellite backhaul costs are justified when their footprint allows them to provide backhaul connectivity for hundreds of BSs. Since our backhaul calculations are based on a backhaul link of $100 \mathrm{Km}$ constituting an integral part of the backbone network, without considering the availability of nearby access BSs, cost comparisons would not be fair to a satellite backhaul scenario.

\section{Fiber Optics:}

This solution consists of laying fiber optic cables throughout the long backhaul distance. Although in some situations it is not feasible (or extremely costly) due to the geographical/terrain considerations (e.g. mountains, lakes, etc.), it remains a possible solution in other scenarios (e.g., plains, desert areas). The main limitation is the cost of civil works, which are generally much more expensive than the cable cost. Although the installation costs can reach 200 USD/meter in dense urban areas [9], they go down to 30 USD/meter in rural areas, as noted in [10]. This clearly plays in favor of fiber deployment when severe geographical constraints do not exist.

\section{Microwave Links:}

This solution consists of placing radio frequency (RF) equipment on towers, such that the backhaul transmissions occur over licensed frequencies. Millimeter wave (mmWave) communications can also be used for backhaul using unlicensed frequencies, but their current equipment costs are higher than microwave. In urban areas, the microwave equipment can be placed on poles or the rooftops of existing buildings. In this case, the pole leasing costs should be taken into account in OPEX calculations [9]. However, in long backhaul stretches traversing non-populated areas in order to 
reach remote rural agglomerations, appropriate towers need to be built. The tower costs should be included in CAPEX, and are estimated at around 50,000 USD/tower [10, 11]. Since microwave frequencies are licensed, appropriate fees should be regularly paid. Instead of considering spectrum costs per capita as in [9], we use a cost per link in line with the cost presented in [6], since the investigated rural scenario corresponds to a sparsely populated area. We analyze the costs for different microwave separation distances between towers: 3,5 , and $10 \mathrm{Km}$. This reflects different deployment conditions depending on geographical and weather constraints.

\section{Terrestrial FSO:}

This solution assumes the deployment of FSO towers to ensure backhaul connectivity. We assume tower costs similar to those of microwave towers. This solution does not involve spectrum licenses since it is based on light transmission. However, it is sensitive to certain weather conditions such as fog, and to alignment errors [12]. Therefore, we investigate different separation between terrestrial FSO towers within the ranges discussed in [12]: $0.5,3$, and $5 \mathrm{Km}$.

\section{Vertical FSO:}

In situations where the erection of towers is not practical (or where it faces security issues such as stealing equipment for terrestrial FSO and microwave links), FSO backhaul communications can be performed using HAPs such as drones or unmanned aerial vehicles (UAVs). Since these devices hover at higher altitude, they are less sensitive to weather conditions (e.g., they fly above fog), they can be separated by longer distances than terrestrial FSO. Thus, we consider distances of 5, 10, and $20 \mathrm{Km}$ between two flying platforms. However, they cost around 50,000 USD per platform, and their operational costs are estimated to 859 USD per flying hour [9]. However, when such devices can be fully solar powered, their operational costs can be significantly reduced. In this paper, we assume a maintenance cost comparable to that of microwave links (considered to be 375 USD/year in [9]) and add an increase of 33\% to account for the additional complexity of the equipment, such that the total becomes 500 USD/year.

\section{Cost ANALysis of RURAL BACKHAUL SOLUTIONS}

This section presents the cost analysis results of the technologies listed in the previous section. The various parameters are listed in Table 2. Fig. 2(a) shows the CAPEX results. Clearly, Terrestrial FSO with 500m separation between towers (to have acceptable performance in case of fog) is the most expensive due to an increase in the number of towers, whereas the fiber optic scenario follows. Fig. 2(b) shows the 1-year OPEX results, with the higher costs incurred in case of Vertical FSO. This is expected due to the high flying costs per hour. To have a better idea on the performance of the other scenarios, we present the same 1-year OPEX results without Vertical FSO in Fig. 2(c). Interestingly, solarpowered Vertical FSO appears to be a cost-efficient solution.

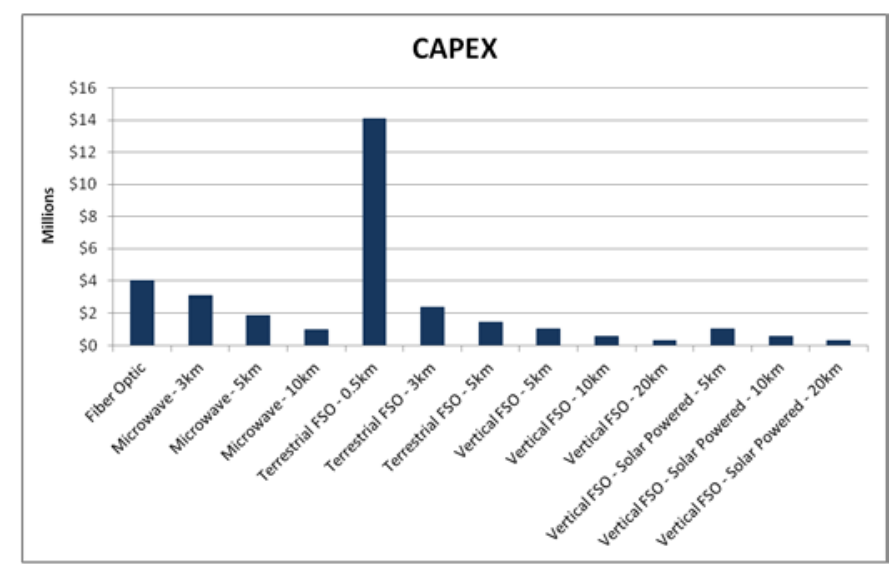

(a)

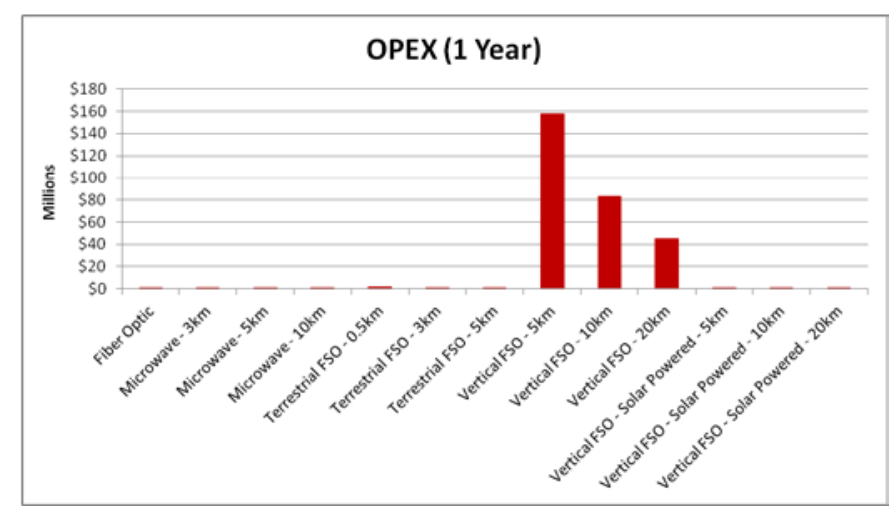

(b)

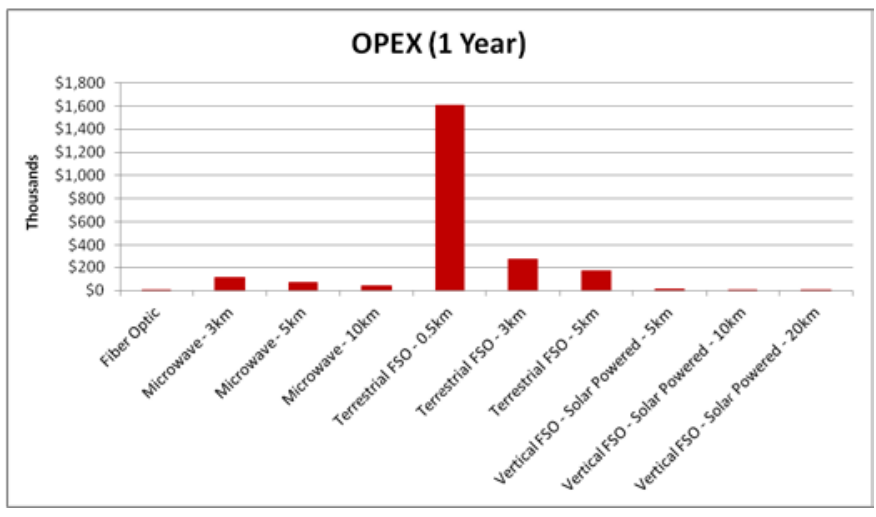

(c)

Figure 2. CAPEX and 1-Year OPEX results: (a) CAPEX results; (b) 1-Year OPEX results; (c) 1-Year OPEX results without Vertical FSO. 
Table 2. Parameter Values used in the Calculations.

\begin{tabular}{|c|c|c|c|}
\hline Parameter & Value & $\begin{array}{c}\text { Type } \\
\text { (CAPEX/ } \\
\text { OPEX) } \\
\end{array}$ & Ref. \\
\hline Total Backhaul Distance (m) & 100,000 & - & - \\
\hline \multicolumn{4}{|c|}{ Microwave } \\
\hline Microwave Tower Cost (USD) & 50,000 & CAPEX & {$[10]$} \\
\hline Microwave Equipment Cost (USD) & 40,000 & CAPEX & {$[10]$} \\
\hline $\begin{array}{l}\text { Spectrum License Costs per Year per Link } \\
\text { (USD) }\end{array}$ & 3,000 & OPEX & {$[6]$} \\
\hline $\begin{array}{l}\text { Microwave Power and Maintenance Cost per } \\
\text { Year (USD) }\end{array}$ & 375 & OPEX & [9] \\
\hline \multicolumn{4}{|c|}{ Fiber } \\
\hline Fiber Cable Cost (USD/m) & 10 & CAPEX & {$[9]$} \\
\hline Fiber Installation Cost (USD/m) & 30 & CAPEX & {$[10]$} \\
\hline Fiber Equipment Cost (USD) & 3,500 & CAPEX & [11] \\
\hline $\begin{array}{l}\text { Fiber Power and Maintenance Cost per Link } \\
\text { per Year (USD) }\end{array}$ & 200 & OPEX & [9] \\
\hline \multicolumn{4}{|c|}{ Terrestrial FSO } \\
\hline Terrestrial FSO Tower Cost (USD) & 50,000 & CAPEX & {$[10]$} \\
\hline Terrestrial FSO Equipment Cost (USD) & 20,000 & CAPEX & [9] \\
\hline $\begin{array}{l}\text { Terrestrial FSO Link Maintenance Cost per } \\
\text { Year (USD) }\end{array}$ & 8,000 & OPEX & [9] \\
\hline \multicolumn{4}{|c|}{ Vertical FSO } \\
\hline Vertical FSO Equipment Cost (USD) & $50,000.00$ & CAPEX & [9] \\
\hline Vertical FSO Operation Cost per Hour (USD) & 859 & OPEX & {$[9]$} \\
\hline Vertical FSO Operation Cost per Year (USD) & $7,524,840$ & OPEX & Calculated from previous row \\
\hline $\begin{array}{l}\text { Vertical FSO Solar Powered Operation Cost } \\
\text { per Year (USD) }\end{array}$ & 500 & OPEX & $\begin{array}{l}\text { Assumed Comparable to } \\
\text { microwave maintenance costs } \\
\text { (with } 33 \% \text { increase) }\end{array}$ \\
\hline
\end{tabular}

\section{Cumulative CAPEX + OPEX Results:}

Fig. 2 showed the CAPEX and 1-year OPEX results. However, to understand the cost-benefit tradeoffs between the various technologies, a longer time visibility is needed. In fact, a relatively high CAPEX technology can outperform another technology with lower CAPEX, if the first technology has a lower OPEX, with savings that accumulate over the years. The non-solar powered Vertical FSO scenarios are orders of magnitude more costly than the other scenarios, which is consistent with the 1-Year results of [9], and with the OPEX results of Fig. 2(b). Hence, the results without Vertical FSO are shown in Fig. 3. Although the costs of fiber deployment appear to be constant, they are increasing slowly with 200 USD OPEX per link per year (as 
indicated in Table 2). The reason is that we have only one link in the studied scenario of $100 \mathrm{Km}$, and thus no repeater maintenance costs. In fact, the distance between repeaters can be shown to be in the order of 350-400 Km [13]. This low OPEX (200 USD per link per year as in Table 2) allows fiber to be competitive in the long run. In fact, Fig. 3 shows that Terrestrial FSO is more cost effective than fiber in the first 6 and 15 years for a tower separation of $3 \mathrm{Km}$ and $5 \mathrm{Km}$, respectively. Afterwards, fiber becomes more cost efficient.

Similarly, Fig. 3 shows that Microwave is more cost effective than fiber in the first 8 and 30 years for a tower

\section{Solar-Powered Vertical FSO - The Loon Project:}

The Loon project, initially launched by Google, consists of launching balloons in the stratosphere to provide connectivity to rural areas [14]. A detailed feasibility study for this project is presented in [15], where the following information is provided:

- $\quad$ Each balloon costs around 17,870 USD, which we will round to 18,000 USD in our calculations (CAPEX).

- Maintenance costs per balloon amount to 1,230 USD each 100 days, which we will round to 5,000 USD/year.

- A balloon covers a diameter of $40 \mathrm{Km}$, which we will reduce to a $33.3 \mathrm{Km}$ distance for FSO transmission, thus requiring three balloons to cover $100 \mathrm{Km}$. To be even more on the conservative side, we will assume that four balloons are needed for covering a $100 \mathrm{Km}$ backhaul link. separation of $3 \mathrm{Km}$ and $5 \mathrm{Km}$, respectively. Afterwards, fiber becomes less costly. Interestingly, solar powered Vertical FSO seems to be very cost efficient on the long run, as it is less costly than fiber even for separation distances of $5 \mathrm{Km}$.

However, solar powered Vertical FSO can be achieved through different techniques, e.g., solar powered drones or balloons. To consider a concrete example with sufficient details in order to investigate further this scenario, we study the special case of Google's Loon project [14] in the next section.

- A balloon's service life is five years. Thus, every five years, we will regenerate CAPEX costs.

The cost results are shown in Fig. 4. Clearly, the Loon project costs are within the range obtained in Fig. 3 for solar powered Vertical FSO. The cost jumps every five years are due to deployment of new balloons.

It should be noted that the cumulative costs in Figs. 3 and 4 are intentionally calculated over an exaggerated period of 40 years, in order to show the CAPEX-OPEX tradeoffs between the various solutions on the long run. In addition, the purpose is to show that even in such an extended operation period with periodically incurred CAPEX costs (as with the Loon case), solar powered Vertical FSO remains cost-effective compared to the other solutions.

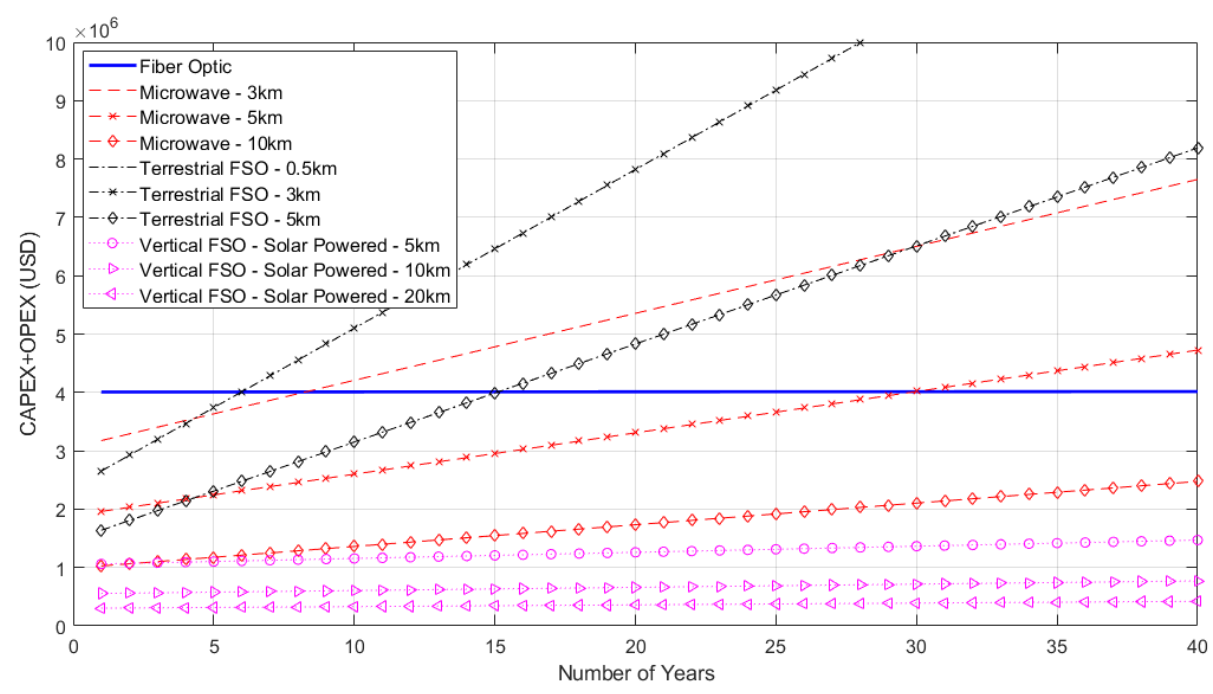

Figure 3. Long-term CAPEX and OPEX results. 


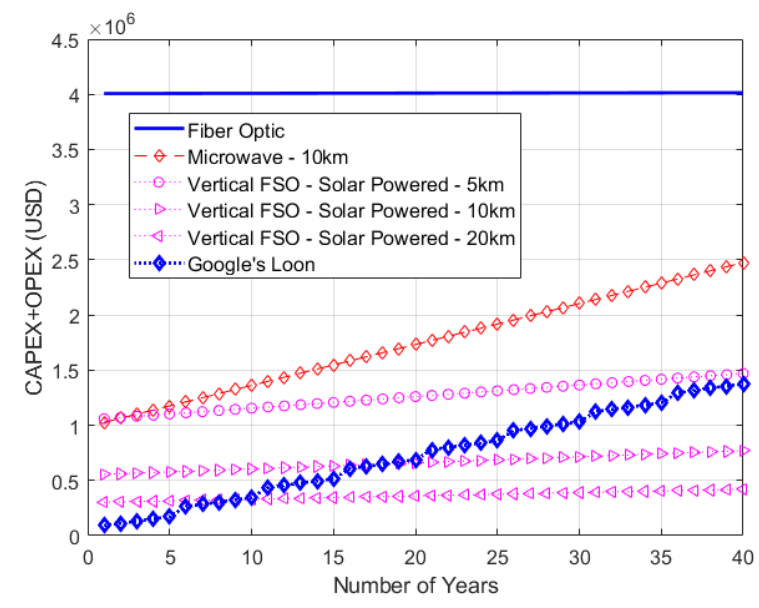

Figure 4. Long-term CAPEX and OPEX results using balloons for solar-powered Vertical FSO.

\section{CONCLUSIONS}

This paper investigated connectivity in rural areas to enable the transition from smart cities to smart living everywhere. Intelligent radio resource management and network planning techniques were discussed for IoT access networks. In addition, several backhauling techniques for providing connectivity to rural areas were discussed and their cost efficiency was analyzed. Techniques based on free space optics with solar powered devices were found to be a suitable solution.

\section{REFERENCES}

[1] S. P. Mohanty, U. Choppali, E. Kougianos, "Everything you wanted to know about smart cities: The Internet of things is the backbone", IEEE Consumer Electronics Magazine, vol. 5 , no. 3, pp. 60-70, July 2016.

[2] E. Yaacoub and Z. Dawy, "On using relays with carrier aggregation for planning 5G networks supporting M2M traffic”, Proceedings of the 10th IEEE International Conference on Wireless and Mobile Computing, Networking and Communications (WiMob 2014), Larnaca, Cyprus, October 2014.

[3] E. Yaacoub, A. Kadri, M. Mushtaha, and A. Abu-Dayya, "Air quality monitoring and analysis in Qatar using a wireless sensor network deployment,” in Proceedings IEEE International Wireless Communications and Mobile Computing Conference (IWCMC 2013), pp. 596-601, Sardinia, Italy, July 2013.

[4] Deloitte, "National transformation in the Middle East: A digital journey", 2017.

[5] L. Chiaraviglio, N. Blefari-Melazzi, W. Liu, J. A. Gutierrez, J. Van De Beek, R. Birke, L. Chen, F. Idzikowski, D. Kilper, P. Monti, and J. Wu, "5G in rural and low-income areas: Are we ready?", 2016 ITU Kaleidoscope: ICTs for a Sustainable World, Bangkok, Thailand, November 2016.

[6] V. Diaz, "Backhauling with fibre?", Fibre Systems, Issue 6, pp. 33-34, Winter 2015.

[7] Terragraph - Virtual Fiber for High Speed Fixed Broadband, White Paper, 2018. Available at: https://terragraph.com/ [Accessed Feb. 25, 2019]
[8] K. Ab-Hamid, C. E. Tan, and S. P. Lau, "Self-sustainable energy efficient long range WiFi network for rural communities", IEEE Globecom 2011 Workshop on Rural CommunicationsTechnologies, Applications, Strategies and Policies (RuralComm 2011), pp. 1050-1055, Houston, TX, USA, December 2011.

[9] M. Alzenad, M. Z. Shakir, H. Yanikomeroglu, and M.-S. Alouini, "FSO-based vertical backhaul/fronthaul framework for 5G+ wireless networks", IEEE Communications Magazine, vol. 56, no. 1, pp. 218-224, January 2018.

[10] Tzvika Naveh, "Mobile backhaul: Fiber vs. microwave - Case study analyzing various backhaul technology strategies", Ceragon White Paper, October 2009.

[11] S. Makovejs, "Wired or wireless?", South Asian Wireless Communications, pp. 23-24, Q4 2012.

[12] O. Hasan and M. Taha, "Optimized FSO system performance over atmospheric turbulence channels with pointing error and weather conditions", Radioengineering, vol. 25, no. 4, pp. 658665, December 2016.

[13] D. Tomar, "Improvement in repeater spacing for fiber optic communication", International Research Journal of Engineering and Technology (IRJET), vol. 4, no. 6, pp. 2038-2042, June 2017.

[14] Loon Project, url: https://loon.co/ [Accessed Jan. 24, 2019]

[15] J. Burr, "The feasibility of Google’s project Loon", Australian National University (ANU) College of Engineering, Tech. report U5350804, 2015.

\section{BIOGRAPHIES}

Elias Yaacoub (S'08, M'10, SM'14) received the B.E. degree in Electrical Engineering from the Lebanese University in 2002, the M.E. degree in Computer and Communications Engineering from the American University of Beirut (AUB) in 2005, and the PhD degree in Electrical and Computer Engineering from AUB in 2010. Between 2010 and 2014, he worked as a Research Scientist/R\&D Expert at the Qatar Mobility Innovations Center (QMIC). Afterwards, he joined Strategic Decisions Group (SDG) where he worked as a Consultant till February 2016. Then he joined the Arab Open University (AOU) an Associate Professor. He is currently an Associate Professor at the Computer Science and Engineering Department at Qatar University. His research interests include Wireless Communications, Antenna Theory, IoT, and Physical Layer Security.

Mohamed-Slim Alouini (S'94, M'98, SM'03, F’09) was born in Tunis, Tunisia. He received the Ph.D. degree in Electrical Engineering from the California Institute of Technology (Caltech), Pasadena, CA, USA, in 1998. He served as a faculty member in the University of Minnesota, Minneapolis, MN, USA, then in the Texas A\&M University at Qatar, Education City, Doha, Qatar before joining King Abdullah University of Science and Technology (KAUST), Thuwal, Makkah Province, Saudi Arabia as a Professor of Electrical Engineering in 2009. At KAUST, Prof. Alouini leads the Communication Theory Lab (ctl.kaust.edu.sa) and his current research interests include the modeling, design, and performance analysis of wireless communication systems. 